\title{
SEMAY OL, NUEVA VARIEDAD DE CÁRTAMO OLEICA
}

\section{SEMAY OL, A NEW OLEIC SAFFLOWER VARIETY}

\section{Alberto Borbón-Gracia ${ }^{1 *}$, Lope Montoya-Coronado', Xochilt M. Ochoa-Espinoza', Néstor A. Aguilera-Molina', Eva Ávila-Casillas ${ }^{2}$ y Carlos I. Cota Barreras ${ }^{3}$}

\author{
IInstituto Nacional de Investigaciones Forestales, Agrícolas y Pecuarias (INIFAP), Campo Experimental Norman E. Borlaug, Ciudad Obregón, Sonora, \\ México. ${ }^{2}$ INIFAP, Campo Experimental Valle de Mexicali, Mexicali, Baja California, México. ${ }^{3}$ INIFAP, Campo Experimental Todos Santos, La Paz, Baja \\ California Sur, México. \\ *Autor para correspondencia (borbon.alberto@inifap.gob.mx)
}

El cártamo (Carthamus tinctorius L.) es una especie oleaginosa que presenta gran adaptabilidad por su rusticidad, bajos requerimientos hídricos (Singh et al., 2016) y pocos problemas de plagas. La planta produce semillas con aceite oleico y linoleico de gran calidad; sin embargo, es un cultivo cuyo potencial de producción no se ha explotado (Ávila et al., 2017). La producción mundial de cártamo en el ciclo 2009-2010 fue de 697 mil toneladas de semilla, la más baja de los últimos siete años. Desde entonces se inició un incremento gradual y en el ciclo 20152016 se produjeron 981 mil toneladas, lo que representa un aumento del $40.7 \%$. En el periodo 2009-2016, México participó con el 16.3 \% de la producción mundial (FAOSTAT, 2018).

En México, el cártamo se produce principalmente en los estados de Sonora, Sinaloa, Baja California y Baja California Sur. Durante los ciclos 2015-2016 y 20162017, la superficie de cártamo fue de 66,406 y 46,095 ha, respectivamente, con un rendimiento medio nacional de $1.72 \mathrm{t} \mathrm{ha}^{-1}$ (SIAP, 2018). En el estado de Sonora se sembró $41 \%$ del área nacional con un rendimiento medio de $2.4 \mathrm{t}$ $\mathrm{ha}^{-1}$, en su mayoría bajo condiciones de riego (SIAP, 2018). En esta región, a partir del ciclo 2000-2001 se presentó la enfermedad falsa cenicilla (Ramularia carthami Z.), que reduce el rendimiento (Hostert et al., 2006).

La liberación de la variedad SEMAY OL, altamente tolerante a falsa cenicilla, permite la siembra de este cultivo con una mayor certidumbre de producción y con un menor costo, ya que no requiere aplicaciones de fungicidas para el control de la enfermedad. Esta variedad es del tipo oleico y proviene de la cruza entre la línea CC1328-1-1-1-3-OY y la variedad CIANO OL, desarrolladas en el Programa de Mejoramiento Genético de Cártamo del CENEB-CIRNO-INIFAP.

La línea CC-1328-1-1-1-3-0Y presenta tolerancia media a roya de la hoja (Puccinia carthami C.) y es altamente tolerante a falsa cenicilla y a tizón de la hoja (Alternaria carthami C.). En cuanto al progenitor masculino, la variedad CIANO OL se caracteriza por su alto número de semillas por capítulo, contenido de aceite y ácido graso oleico.

En la generación $F_{1}$ se aplicó selección masal y selección individual en la $F_{2}$. La selección en masa para obtener la línea avanzada se llevó a cabo en la generación $F_{3}$. A partir de $\mathrm{F}_{4}$ la línea experimental CC1572-1-1-OY se evaluó en ensayos de rendimiento. Las selecciones individuales y en masa se llevaron a cabo en el Valle del Yaqui, Sonora $\left(27^{\circ}\right.$ $22^{\prime} 14.39^{\prime \prime}$ latitud N, $109^{\circ} 55^{\prime} 52.40^{\prime \prime}$ longitud O y 40 m de altitud).

SEMAY OL presenta en promedio $115 \mathrm{~cm}$ de altura, su hábito de crecimiento es determinado y erecto; su floración ocurre 101 días después de la siembra y a los 157 d la semilla alcanza su madurez fisiológica. Las hojas son de color verde claro, de forma ovoide con bordes aserrados. El tallo es erecto, sólido y resistente al acame (Figura 1). Antes de la floración, el capítulo es de forma cónica y completamente cubierto por las brácteas; produce 32 capítulos por planta, con un promedio de 46 granos por capítulo. Las flores frescas son de color amarillo y cuando se secan se tornan anaranjadas (Figura 2); el polen es de color amarillo. Las semillas son de forma oval, color café amarillento y longitud promedio de $7.0 \mathrm{~mm}$ y $2.0 \mathrm{~mm}$ de ancho, con $41 \%$ de pericarpio y $59 \%$ de almendra; carece de vilano. El peso promedio de 1000 semillas es de $34.0 \mathrm{~g}$ con un peso específico de $52.0 \mathrm{~kg} \mathrm{hL}^{-1}$. La semilla contiene, en promedio, $36 \%$ de aceite y $20 \%$ de proteína. El aceite es de tipo oleico, con un promedio de $79.8 \%$ de ácido oleico y $12.1 \%$ de ácido linoleico.

En los Valles del Yaqui y del Mayo, Sonora el rendimiento de semilla de la variedad SEMAY OL, bajo presión de falsa cenicilla y sin aplicaciones de fungicidas para el control 


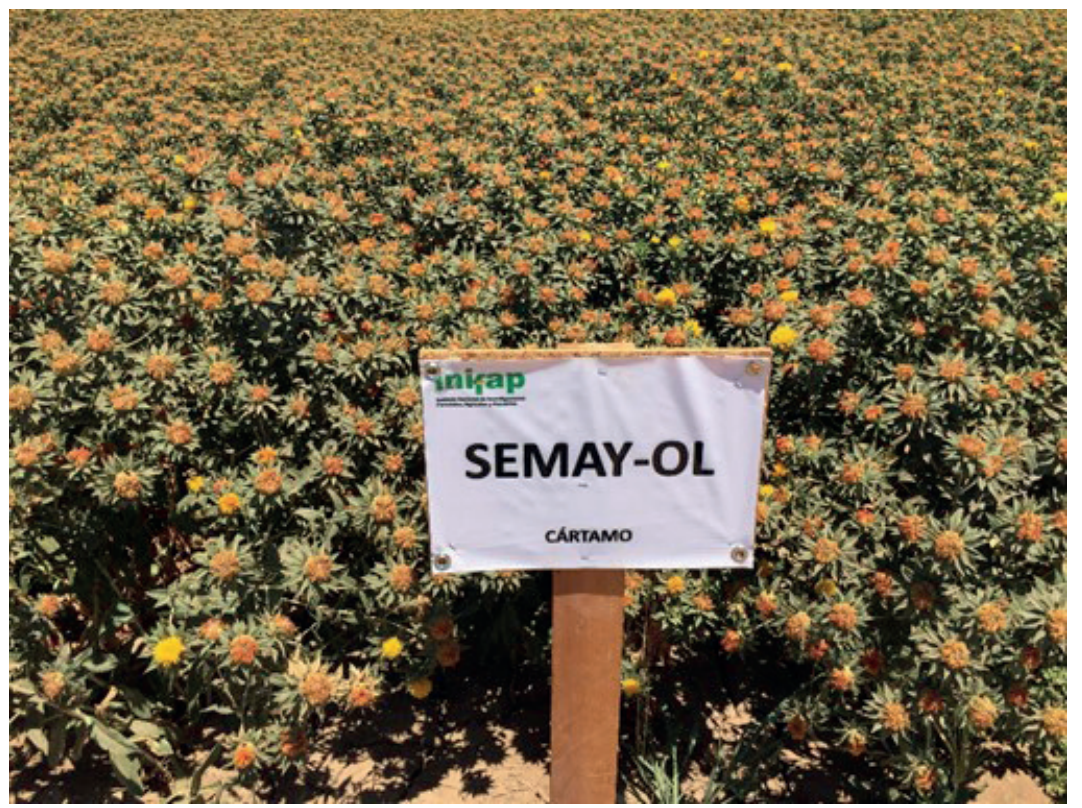

Figura 1. Aspecto general de las plantas de la variedad de cártamo SEMAY OL en campo.
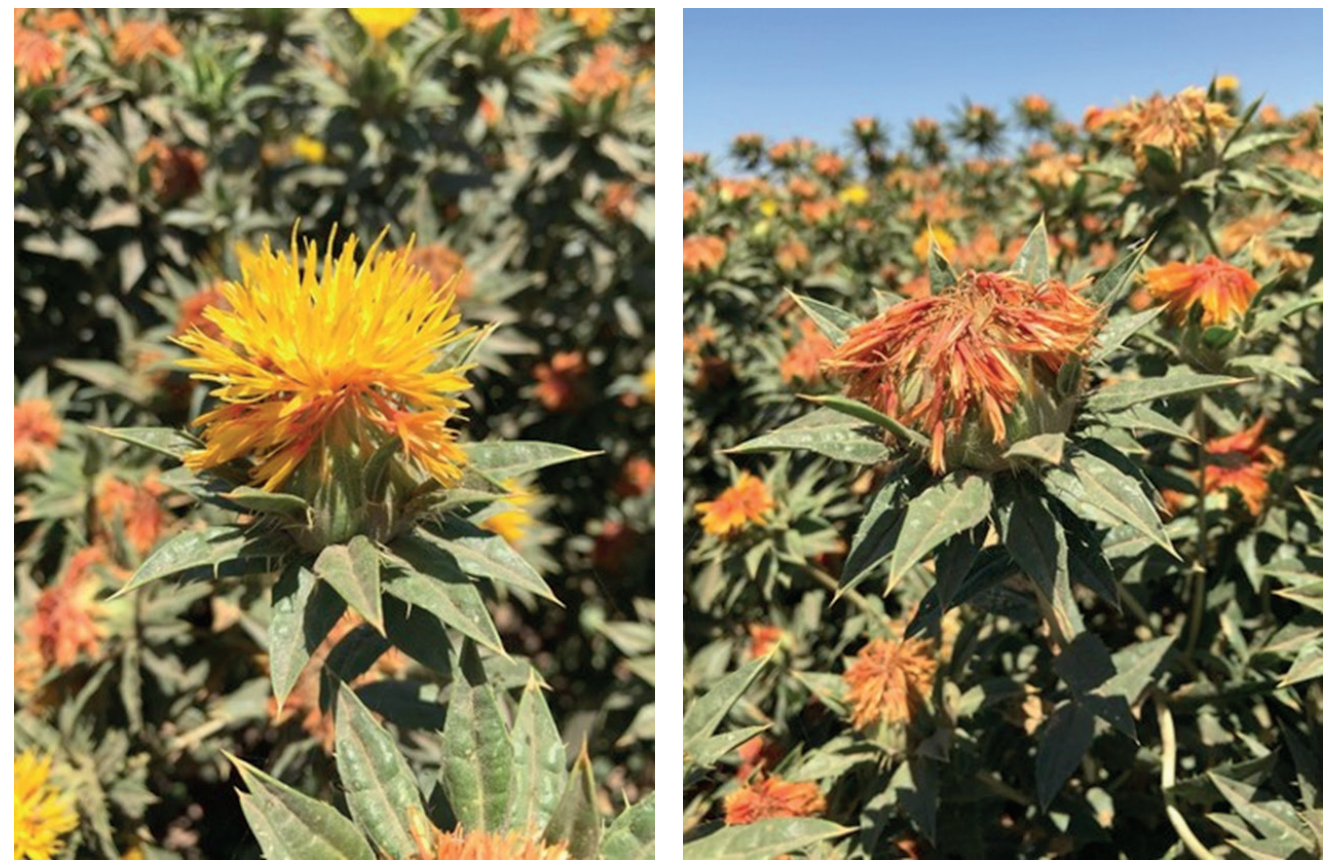

Figura 2. Aspecto de los capítulos frecos (A) y secos (B) de la variedad de cártamo SEMAY OL

de la misma, en promedio de tres ciclos agrícolas (20142015 a 2016-2017) fue de $2125 \mathrm{~kg} \mathrm{ha}^{-1}$, superior en $12 \%$ a la variedad comercial testigo CIANO OL. Esta variedad se recomienda para el ciclo de producción otoño-invierno en los estados de Sonora, Sinaloa, Baja California y Baja California Sur.

La variedad SEMAY OL se registró con el número CAR-023-091318 en el Catálogo Nacional de Variedades
Vegetales del Servicio Nacional de Inspección y Certificación de Semillas (SNICS). La semilla básica está a disposición de los agricultores del noroeste de México en el CENEB-INIFAP, en Ciudad Obregón, Sonora.

\section{AGRADECIMIENTOS}

Al Instituto Nacional de Investigaciones Forestales Agrícolas y Pecuarias (INIFAP), a la Secretaría de Agricultura, 
Ganadería, Desarrollo Rural, Pesca y Alimentación (SAGARPA, actualmente SADER), a la Fundación PIEAES de Sonora, México, A. C. y al Patronato para la Investigación y Experimentación Agrícola en el estado de Sonora (PIEAES), por el financiamiento de los trabajos de investigación que condujeron a la obtención de la variedad SEMAY OL.

\section{BIBLIOGRAFÍA}

Ávila C. E., X. M. Ocho E., L. Montoya C., N. A. Aguilera M., A. Borbón G. y J. I. Alvarado P. (2017) Chey-ol: nueva variedad de cártamo oleica para el noroeste de México. Revista Mexicana de Ciencias Agrícolas 8:1209-1212.

FAOSTAT, Food and Agriculture Organization of the United Nations (2018)
Crops. Food and Agriculture Organization of the United Nations. Rome, Italy. http://www.fao.org/faostat/en/\#data/QC (Diciembre 2018).

Hostert N. D., C. L. Blomquist, S. L. Thomas, D. G. Fogle and R. M. Davis (2006) First report of Ramularia carthami, causal agent of Ramularia leaf spot of safflower, in California. Plant Disease 90:1260, https://doi.org/10.1094/PD-90-1260C

SIAP, Servicio de Información Agroalimentaria y Pesquera (2018) Anuario estadístico de la producción agrícola. Servicio de Información Agroalimentaria y Pesquera, Secretaría de Agricultura y Desarrollo Rural. Ciudad de México. https://nube.siap.gob.mx/ cierreagricola/ (Diciembre 2018).

Singh S., S. V. Angadi, R. St. Hilaire, K. Grover and D. M. VanLeeuwen (2016) Spring safflower performance under growth stage based irrigation in the Southern high plains. Crop Science. 56:18781889, https://doi.org/10.2135/cropsci2015.08.0481 